\title{
Two Step on-axis Digital Holography Using Dual-channel Mach-Zehnder Interferometer and Matched Filter Algorithm
}

\author{
Hyung-Chul Lee and Soo-Hyun Kim* \\ Department of Mechanical Engineering, Korea Advanced Institute of Science and \\ Technology, 373-1 Kusong-dong, Yusong-gu, Daejeon 305-701, Korea \\ Dae-Suk Kim \\ Division of Mechanical System Engineering, Chonbuk National University, \\ 664-14 Duckjin-dong, Duckjin-gu, Jeonju 561-756, Korea
}

(Received July 19, 2010 : revised September 28, 2010 : accepted September 30, 2010)

\begin{abstract}
A new two step on-axis digital holography (DH) is proposed without any assumptions, phase shifting, or complicated optical components. A dual-channel Mach-Zehnder interferometer was employed. Using that setup, the object field can be reconstructed requiring only two step measurements. To eliminate position difference between two charge-coupled device (CCD) cameras, a matched filter algorithm was used. Experimental results are compared to those of the traditional phase shifting technique. The proposed approach can also be applied to single-exposure on-axis $\mathrm{DH}$ for real time measurement.
\end{abstract}

Keywords: Digital holography, Mach-Zehnder interferometer, Matched filter

OCIS codes : (090.2880) Holographic interferometry; (000.3110) Instruments, apparatus, and components common to the sciences

\section{INTRODUCTION}

$\mathrm{DH}$ is a novel technique for recording and reconstructing the fully complex field of a wavefront [1-2]. Two processes are involved in $\mathrm{DH}$ : the recording process, and the hologram reconstruction process. Due to the nature of holography, three diffraction components: the dc term, object field and its conjugate, are produced in the reconstruction process. In off-axis DH, these three diffraction components are spatially separated. In contrast, in on-axis $\mathrm{DH}$, the diffraction components are overlapped. However, on-axis DH has a larger field of view and space bandwidth product (SBP) than off-axis $\mathrm{DH}$, features that could be very useful in some applications [3]. To solve the overlap problem of on-axis $\mathrm{DH}$, various efforts have been made.

The use of multiple exposures with phase shifting is a simple solution and could eliminate both the de term and conjugate field [4-5]. However, it requires a phase shifting mechanism during measurements. Maleki and Devaney suggested the recording of two holograms at two different distances [6]. By processing the holograms in the spatial frequency domain, they extracted the object image without the conjugate image. However, because singularities occur during the computation, they had to use an inverse filter and regularization, which requires more extensive computation. Kim and Javidi [7] also used two holograms. To reduce the dc term, the average intensity of the hologram was subtracted from the hologram. However, this method is limited to an object which has a uniform intensity distribution. Situ [8] reported a method which records four holograms from two recording planes. This method still requires a phase delay of reference beams during measurements, and it has a singularity problem.

Zhang and Tiziani [9-10] assumed a weak object wave so that the hologram signal could be approximated as an exponential function. Although this method involves simple computation and does not suffer from singularities, the assumption of the weak object wave is not always valid and favorable in terms of the signal to noise ratio. Liu and Poon [11] also assumed a weak object wave, although they

\footnotetext{
*Corresponding author: soohyun@kaist.ac.kr

Color versions of one or more of the figures in this paper are available online.
} 
retrieved the object field by two step measurements.

There are other purely iterative methods that use the Gerchberg-Saxton algorithm [12-13]. These methods, however, may strongly depend on initial guesswork and requires computations to obtain an optimized result.

Another approach using a tilted reference wave was proposed [14-16]. It has a very simple optical setup and requires no phase shifting devices. However, the isotropic phase shifting is not possible in that method. As a phaseshifting free method like the tilted reference approach, a single-exposure technique using fractional Talbot effect was reported [17]. However, the accurate fabrication and alignment of the gratings are difficult, and have a great effect on the resultant image.

Recently, pixelated optical components have been used [18-22]. These approaches can directly record phase-shifted holograms with specially designed components. However, they are difficult to fabricate and align. As a related work, Nomura [23] suggested a method using a random-phase reference wave. This method requires only ordinary imaging devices. However, they assumedthe same object and reference intensity on any neighboring four pixels.

In this paper, a new two step on-axis DH is proposed. Unlike the above mentioned methods, it does not require any phase shifting during the recording process, weak object wave assumption, and any other complicated optical components. The proposed method also provides a simple reconstruction algorithm and system setup.

A dual-channel Mach-Zehnder interferometer was employed to verify our method. The matched filter algorithm [24] was also employed to align two CCDs. Simulation and experiment results are presented as a demonstration.

\section{BASIC PRINCIPLE}

FIG. 1. is the schematic diagram of the experimental setup. The light source is a single-mode laser with $635 \mathrm{~nm}$ wavelength. Laser light is collimated by a spatial filter and lens, and then passes through a linear polarizer (P3) whose polarization direction is $45^{0}$ with respect to the $\mathrm{x}$-axis. This beam is then divided by a beam splitter (BS1) into two paths, one of which illuminates the transparent object, and the other, which passes through a quarter-wave plate, acts as the reference beam. The quarter-wave plate has the fast-axis along the $y$-axis. Therefore, $\pi / 2$ phase shift occurs only on the $\mathrm{x}$-axis component $\left(0^{0}\right)$ of reference beam. Using two polarizers $(\mathrm{P} 1, \mathrm{P} 2)$ whose polarization directions are $90^{\circ}, 0^{\circ}$ respectively, we can make a $\pi / 2$ phase-shifted reference wave on CCD2 with respect to that of the CCD1. That makes it possible to obtain two holograms simultaneously with $\pi / 2$ phase shift between them.

Generally, in on-axis DH, the hologram can be represented as follows,

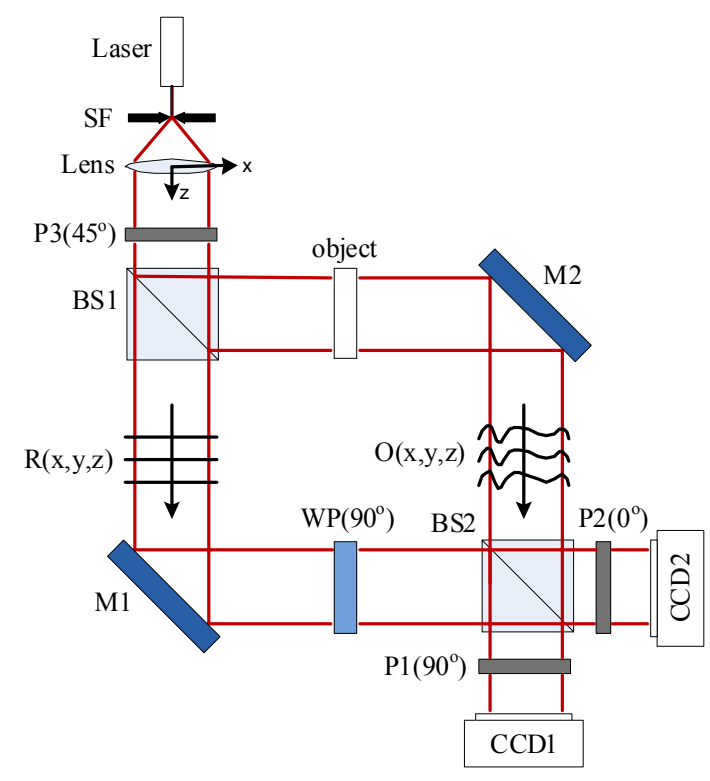

FIG. 1. Dual-channel Mach-Zehnder interferometer with a quarter-wave plate. SF, spatial filter; P, polarizer; BS, beam splitter; M, mirror; WP, wave plate.

$$
\begin{aligned}
& H(x, y, \theta)=|O(x, y)|^{2}+|R(x, y)|^{2} \\
& +O(x, y) R^{*}(x, y) \exp (-j \theta)+O^{*}(x, y) R(x, y) \exp (j \theta)
\end{aligned}
$$

where, $\mathrm{x}, \mathrm{y}$ are the rectangular coordinates of the recording plane, $\theta$ is the phase shift, $\mathrm{O}(\mathrm{x}, \mathrm{y}), \mathrm{R}(\mathrm{x}, \mathrm{y})$ are the object and reference field, and * denotes the complex conjugate. To retrieve the object field, a two hologram method [7] was chosen among many reconstruction approaches. The method consists of two procedures. First, a complex field is obtained at the recording plane using Eq. (2),

$$
\begin{aligned}
u_{r}(x, y)= & O(x, y) R^{*}(x, y) \\
= & \frac{1}{2}\left\{\left[H(x, y, 0)-|O(x, y)|^{2}-|R(x, y)|^{2}\right]+j\left[H\left(x, y, \frac{\pi}{2}\right)-\right.\right. \\
& \left.\left.|O(x, y)|^{2}-|R(x, y)|^{2}\right]\right\}
\end{aligned}
$$

where, $H(x, y, 0), H(x, y, \pi / 2)$ are the hologram and its $\pi / 2$ phase-shifted one. Second, object field is reconstructed by the Fresnel transformation,

$$
\begin{array}{r}
u_{o}\left(x^{\prime}, y^{\prime}\right)=\frac{\exp (j k d)}{j d \lambda} \exp \left[\frac{j k}{2 d}\left(x^{\prime 2}+y^{\prime 2}\right)\right] \\
F\left\{u_{r}(x, y) \exp \left[\frac{j k}{2 d}\left(x^{2}+y^{2}\right)\right]\right\}
\end{array}
$$

where, $x^{\prime}, y^{\prime}$ are the rectangular coordinates of the reconstruction plane, $\mathrm{k}$ is the wavenumber, $\mathrm{d}$ is the distance between recording and reconstruction planes, $\lambda$ is the wavelength of the light, and $F$ denotes the two dimensional fast Fourier transformation. 
Note that the object field can be obtained by two step measurements, if we use the Mach-Zehnder interferometer as shown in the FIG. 1. First, two holograms can be obtained simultaneously, having a $\pi / 2$ phase shift between them. Second, object intensity can be measured by blocking the reference arm. Substituting these two holograms and the object intensity into Eq. (2), and then using Eq. (3), the object field can be reconstructed. Here, note that reference intensity is constant in time. Therefore, there is no necessity for measuring reference intensity each time.

\section{ANALYSIS AND RESULT}

However, if the two CCDs have different positions relative to the object, the position effect should also be considered in the two hologram approach. For example, a $\mathrm{CCD}$ can have position information relative to the object, which are $\mathrm{x}, \mathrm{y}, \mathrm{z}$, roll, pitch, and yaw, as shown in the FIG. 2. In general, the position information of two CCDs are different from each other. Therefore, a resolution target was employed as an object as shown in the FIG. 3(a) to examine the effect of position difference between two CCDs on the reconstructed image.

If the two CCDs have the same position relative to the object, the two holograms obtained using FIG. 1 have a difference of $\pi / 2$ phase shift between them. Therefore, the object field is reconstructed without any noise components as shown in the FIG. 3(b). However, if there is any difference between position information of the two CCDs, we cannot retrieve a clean object image as shown in the FIG. 3(c) and (d). FIG. 3(c) was obtained when there was a lateral shift of two pixels between two CCDs in both $\mathrm{x}$ and $y$ directions. And, FIG. 3(d) was obtained when an axial shift of $500 \mu \mathrm{m}$ existed between two CCDs in the $\mathrm{z}$ axis. As you can see in the FIG. 3, a small position difference can badly influence the reconstructed image. Therefore, it is in practice important that two CCDs be aligned each other to have the same positions relative to the object, especially before any measurements.

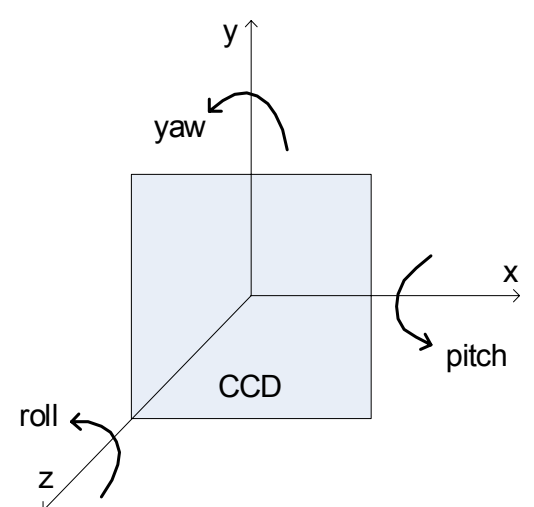

FIG. 2. Six geometric variables: $x, y, z$, roll, pitch, and yaw.
In the previous paper [24], we proposed an alignment method between two CCDs using a matched filter algorithm. To put it simply, the algorithm is a kind of correlation method, which compares intensities of two CCDs. When two CCDs have the same position for an object, their intensity patterns may be very similar. Therefore, the correlation between the intensities is almost one. On the other hand, when the two CCDs have different positions, the correlation between the intensities may be a value lower than one. Therefore, in the process of making the correlation approach one, the two CCDs should have the same position for an object. For detailed descriptions about matched filter algorithm, refer to the previous paper.

FIG. 4 (a) is the reconstructed object intensity obtained

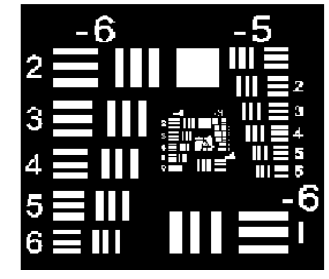

(a)

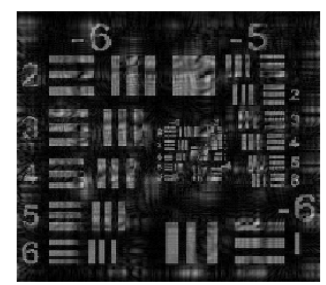

(c)

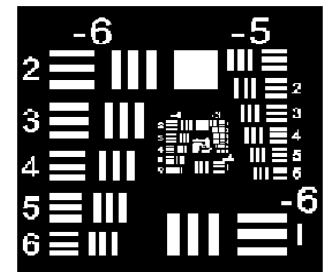

(b)

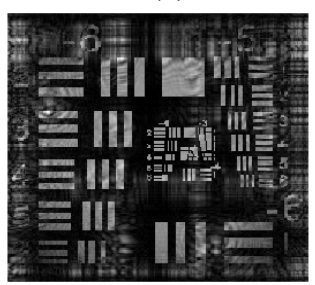

(d)
FIG. 3. Simulation result: (a) original object, (b) reconstructed object without position error, (c) reconstructed object with lateral position error, (d) reconstructed object with axial position error.
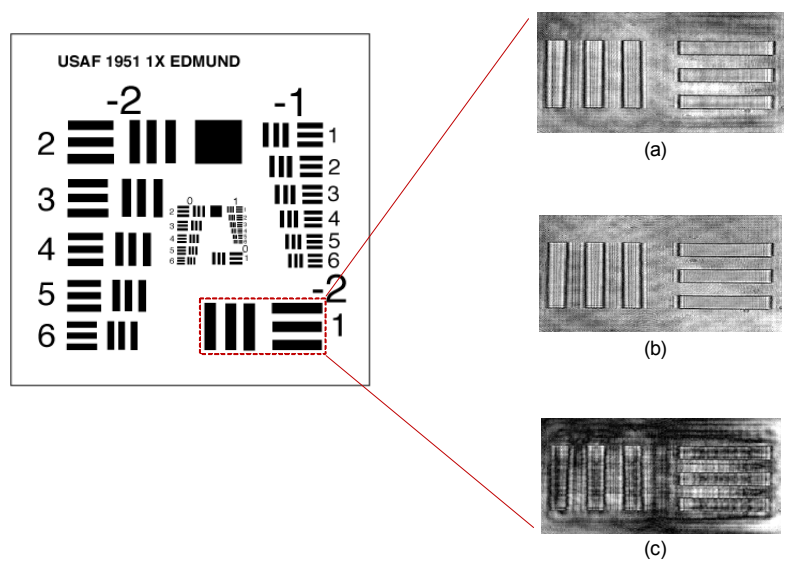

FIG. 4. Experimental result: (a) proposed method, (b) conventional phase-shifting method, (c) on-axis $\mathrm{DH}$ without phase shifting. 


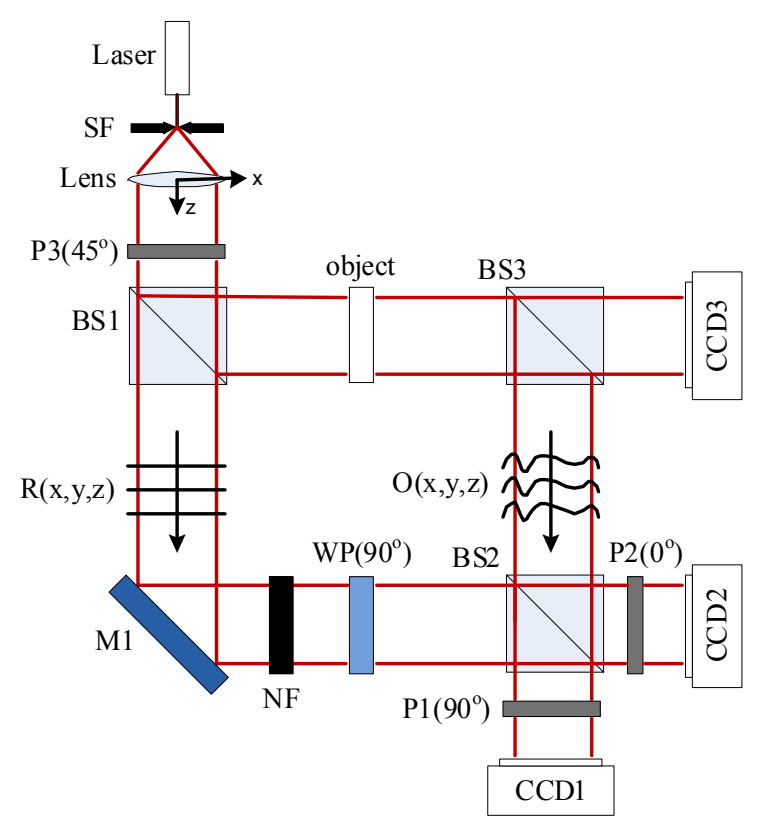

FIG. 5. Three-channel Mach-Zehnder interferometer for single-exposure DH. NF, neutral density filter.

by the proposed dual-channel on-axis DH. A part of USAF resolution target (38-257, Edmund) was employed as an object as shown in the FIG. 4. FIG. 4(b) is reconstructed by the conventional phase shifting method which has only one CCD. Therefore, there are not any noises caused by the position difference between detectors. FIG. 4(c) is obtained by one-channel on-axis $\mathrm{DH}$ without any phase shifting process. As you can see, the conjugate component of the object degrades the reconstructed image.

\section{DISCUSSION}

As shown in the FIG. 4, the proposed dual-channel on-axis DH method provides almost the same quality result compared to the conventional phase shifting scheme. Therefore, we can retrieve the object field much faster than by conventional approaches, if the position difference between two CCDs is eliminated. The proposed method consists of two processes: measurement of two phaseshifted holograms and the object intensity. In addition, there are not any limitations in our method.

Furthermore, single-exposure on-axis DH can be realized in practice based on our scheme. To realize single-exposure on-axis DH, we should use one more channel as shown in the FIG. 5. FIG. 5 is almost identical to FIG. 1, except the added detector, CCD3. CCD3 measures object intensity, and $\mathrm{CCD} 1$ and 2 measure two phase-shifted holograms. Then, using Eq. (2), (3), single-exposure on-axis DH can be realized in practice without any limitations. The neutral density filter decreases the reference intensity approximately in half to match the intensity level of reference and object.

\section{CONCLUSION}

In this paper, a new two step on-axis DH was proposed. A dual-channel Mach-Zehnder interferometer was employed to implement the scheme. Object field was retrieved in two step measurements. We eliminated position differences between two CCDs using matched filter algorithm, and then provided a reconstruction image which was of almost the same quality as the result from the conventional phase-shifting method. In addition, there are not any limitations in our method. Furthermore, if we use one more channel, single-exposure on-axis $\mathrm{DH}$ can be realized in a practical way. We believe that our scheme can be widely applied to real-time applications like monitoring dynamics of biological samples or particles in flows.

\section{ACKNOWLEDGMENT}

This work was supported by the National Research Foundation of Korea (NRF) grant funded by the Korean government (MEST) (2010-0016064).

\section{REFERENCES}

1. U. Schnars and W. Juptner, "Direct recording of holograms by a CCD target and numerical reconstruction," Appl. Opt. 33, 179-181 (1994).

2. U. Schnars and W. Juptner, "Digital recording and numerical reconstruction of holograms," Meas. Sci. Technol. 13, R85 (2002).

3. A. Stern and B. Javidi, "Theoretical analysis of three-dimensional imaging and recognition of micro-organisms with a singleexposure on-line holographic microscope," J. Opt. Soc. Am. A 24, 163-168 (2007).

4. Y. Takaki, H. Kawai, and H. Ohzu, "Hybrid holographic microscopy free of conjugate and zero-order images," Appl. Opt. 38, 4990-4996 (1999).

5. I. Zhang, "Phase-shifting digital holography," Opt. Lett. 22, 1268-1270 (1997).

6. M. Maleki and A. Devaney, "Noniterative reconstruction of complex-valued objects from two intensity measurements," Opt. Eng. 33, 3243-3253 (1994).

7. D. Kim and B. Javidi, "3D object recognition using single exposure on-line digital holography," Proc. SPIE 6027, 602703-602709 (2006).

8. G. Situ, J. Ryle, U. Gopinathan, and J. Sheridan, "Generalized in-line digital holographic technique based on intensity measurements at two different planes," Appl. Opt. 47, 711-717 (2008).

9. Y. Zhang and X. Zhang, "Reconstruction of a complex object from two in-line holograms," Opt. Express 11, 572578 (2003).

10. Y. Zhang, G. Pedrini, W. Osten, and H. Tiziani, "Reconstruction of in-line digital holograms from two intensity measurements," Opt. Lett. 29, 1787-1789 (2004).

11. J. Liu and T. Poon, "Two-step-only quadrature phase-shifting 
digital holography," Opt. Lett. 34, 250-252 (2009).

12. Y. Zhang, G. Pedrini, W. Osten, and H. Tiziani, "Whole optical wave field reconstruction from double or multi inline holograms by phase retrieval algorithm," Opt. Express 11, 3234-3241 (2003).

13. T. Latychevskaia and H. Fink, "Solution to the twin image problem in holography," Phys. Rev. Lett. 98, 233901 (2007).

14. S. Nakadate and M. Isshiki, "Real-time vibration measurement by a spatial phase-shifting technique with a tilted holographic interferogram," Appl. Opt. 36, 281-284 (1997).

15. Y. Yasuno, S. Makita, T. Endo, G. Aoki, H. Sumimura, M. Itoh, and T. Yatagai, "One-shot-phase-shifting Fourier domain optical coherence tomography by reference wavefront tilting," Opt. Express 12, 6184-6191 (2004).

16. H. Toge, H. Fujiwara, and K. Sato, "One-shot digital holography for recording color 3-D images," Proc. SPIE 6912, 69120U (2008)

17. L. Martinez-Leon, M. Araiza-E, B. Javidi, P. Andres, V. Climent, J. Lancis, and E. Tajahuerce, "Single-shot digital holography by use of the fractional Talbot effect," Opt. Express 17, 12900-12909 (2009).

18. Y. Awatsuji, M. Sasada, and T. Kubota, "Parallel quasi- phase-shifting digital holography," Appl. Phys. Lett. 85, 1069 (2004).

19. Y. Awatsuji, A. Fujii, T. Kubota, and O. Matoba, "Parallel three-step phase-shifting digital holography," Appl. Opt. 45, 2995-3002 (2006).

20. Y. Awatsuji, T. Koyama, T. Tahara, K. Ito, Y. Shimozato, A. Kaneko, K. Nishio, S. Ura, T. Kubota, and O. Matoba, "Parallel optical-path-length-shifting digital holography," Appl. Opt. 48, 160-167 (2009).

21. T. Nomura, S. Murata, E. Nitanai, and T. Numata, "Phaseshifting digital holography with a phase difference between orthogonal polarizations," Appl. Opt. 45, 4873-4877 (2006).

22. H. Suzuki, T. Nomura, E. Nitanai, and T. Numata, "Dynamic recording of a digital hologram with single exposure by a wave-splitting phase-shifting method," Opt. Rev. 17, 176180 (2010).

23. T. Nomura and M. Imbe, "Single-exposure phase-shifting digital holography using a random-phase reference wave," Opt. Lett. 35, 2281-2283 (2010).

24. H. Lee, D. Kim, and S. Kim, "A simple and quantitative alignment procedure between solid state cameras," Opt. Express 17, 23947-23952 (2009). 